\title{
Exploración de atributos de valor percibido en operadores de telefonía móvil. Características y relaciones
}

FECHA DE RECEPCIÓN: 17 de enero FECHA DE APROBACIÓN: 1 de febrero Pp. 27-40

\section{Resumen}

El valor percibido es uno de los conceptos más importantes en la literatura del marketing, especialmente en las relaciones que mantienen los consumidores con las marcas y empresas, que para el presente artículo, pertenecen a la industria de las telecomunicaciones. El objetivo del estudio está relacionado con la exploración de los atributos que intervienen en el proceso de percepción de valor de los operadores telefónicos, con el fin de conocer las características más importantes que pueden influenciar la preferencia y sostenimiento del servicio. Se utilizó un diseño descriptivo relacional que involucró a 376 personas. Los resultados aportan evidencias en cuanto a que las empresas deben construir y mantener vínculos simbólicos que refuercen su propuesta de valor de marketing incluyendo el ámbito digital.

\section{Palabras clave}

Valor percibido, telefonía móvil, operadores de telefonía móvil.

\section{Leonardo Ortegón Cortázar}

Magíster en Psicología del Consumidor, profesor de la maestría en Gerencia Estratégica de Mercadeo. Institución Universitaria Politécnico, Grancolombiano. Autor de correspondencia.

\section{Milton Wilches Tamayo}

Magíster en Gerencia Estratégica de Mercadeo, Institución Universitaria Politécnico Grancolombiano; profesional en Publicidad; profesor universitario de la Corporación Universitaria Unitec e Institución Universitaria Los Libertadores.

\section{Nadia Andrea Soledad Rojas}

Magíster en Gerencia Estratégica de Mercadeo, Institución Universitaria Politécnico Grancolombiano; Comunicadora Social y Periodista, Universidad Sergio Arboleda.

\section{Deiby Alejandro Ayala}

Magíster en Gerencia Estratégica de Mercadeo,Institución Universitaria Politécnico Grancolombiano; profesional en Mercadeo y Publicidad, Institución Universitaria Politécnico Grancolombiano. 


\title{
Exploration of Perceived Value Attributes in Mobile Telephone operators. Features and Relations
}

\begin{abstract}
Abstrac. Perceived value is one of the most outstanding concepts in the literature review of marketing, emphasizing the relationship that consumers have with brands and companies belonging to the telecommunication industry. This study aims at exploring the attributes which intervene in the perception process of mobile telephone operators to know the most important features which influence preference and sustainability of services. A relational descriptive design involving 376 people was applied. Its outcomes show that these companies should build and keep symbolic ties which really enhance the proposal of marketing added value including digital environments.
\end{abstract}

Key words. Perceived value, mobile telephony, mobile telephone operators.

\section{Exploration d'attributs de valeur perçus par les opérateurs de téléphonie mobile : caractéristiques et relations.}

Resumé. La valeur perçue est l'un des concepts les plus importants de la littérature marketing lorsqu'il s'agit des relations que les consommateurs entretiennent avec les marques et les entreprises qui, le cas échéant, appartiennent à l'industrie des télécommunications. L'objectif de cette étude consiste à explorer les facteurs intervenant lors du processus de perception de valeur des opérateurs téléphoniques de façon à connaître les caractéristiques les plus importantes pouvant influencer la préférence et la continuité du service. Une analyse de type descriptivo-relationnelle a été mise en place impliquant la participation de 376 personnes. Les résultats mettent en évidence que les entreprises doivent construire et maintenir les liens symboliques renforçant leur proposition de valeur-marketing incluant le domaine digital.

Mots clefs. Valeur perçue, téléphonie mobile, opérateurs de téléphonie mobile

\section{Exploração de Atributos de valor percebido em operadoras de telefonia móvel. Características e relações}

Resumo. O valor percebido é um dos conceitos mais importantes na literatura de marketing, especialmente nas relações que os consumidores mantêm com marcas e empresas, que nesse estudo pertencem aos conceitos da indústria de telecomunicações. O objetivo do estudo foi explorar os atributos envolvidos no processo de percepção de valor das operadoras de telefonia a fim de conhecer as características mais importantes que podem influenciar a preferência e a fidelidade do cliente em relação a um determinado serviço. Foi utilizado um desenho descritivo relacional que envolveu 376 pessoas. Os resultados fornecem evidências de que as empresas devem construir e manter links simbólicos para reforçar a sua proposta de valor de marketing, incluindo o âmbito digital.

Palabras chave. Valor percebido, Telefonia Móvel, Operadoras de telefonia móvel. 


\section{Introducción}

$\mathbf{E}$ I auge de la telefonía celular en Colombia y su penetración en el territorio nacional y el desarrollo tecnológico de los últimos años, hacen de este sector uno de los de mayor crecimiento en el país (Gómez, Polo y Rivera, 2011). Además la democratización del uso de teléfonos móviles, a nivel mundial, es un propósito de todas las naciones según lo explican Vargas et al., (2012 p. 49) estando asociado a uno de los objetivos del milenio sugeridos por las Naciones Unidas.

En los últimos años, la telefonía móvil ha sido la tecnología de más rápido desarrollo de la historia. Se estima que en la actualidad existen más de 6.915 millones de suscripciones a telefonía móvil en el mundo (International Telecommunication Union, 2014). Lo anterior demuestra una rápida apropiación de nuevas tecnologías en Colombia, que la convierten en uno de los países que más ha asimilado esta tecnología (Revista Dinero, 2013) especialmente en la activación de teléfonos inteligentes.

En el ámbito de los operadores móviles, existe una batalla por los mercados, según lo expresan Vargas et al., (2012), en donde cada vez es más difícil para una compañía de este sector poder mantener a sus clientes; esto se debe al impacto de la tecnología sobre la calidad y la satisfacción. Lo anterior es constatado por Cerezo (2008), quien describe que la primera década del siglo ha estado marcada por la consolidación de la movilidad como medio de comunicación, logrando que los smartphone se presenten como el nuevo motor de la sociedad de la información.

Por lo anterior, la masificación del acceso a dispositivos móviles y la explosión en el desarrollo de tecnologías y aplicaciones ligadas al uso del teléfono celular, han influido en el proceso de cambio generacional y cultural en todo el mundo (Martínez y Jacobo, 2014) al que no es ajeno el mercado colombiano.

Más que un simple medio de comunicación, la telefonía celular se ha convertido en un elemento crucial en el estilo de vida del consumidor, determinando en gran medida, la forma en que los consumidores son productivos, se entretienen y se relacionan entre sí frente a un amplio número de intereses (Ruelas, 2010), incitando la necesidad de analizar dichas variables con relación a la percepción de los operadores de telefonía móvil. Así, el principal objetivo de esta investigación fue explorar los atributos que intervienen en el proceso de percepción de valor de los operadores telefónicos, a fin de conocer las características más importantes que pueden influenciar la preferencia y sostenimiento del servicio. Para corresponder a este propósito, a continuación se expone una revisión de literatura asociada al concepto de valor percibido y una descripción del mercado de los operadores móviles en Colombia; posteriormente, se presenta la estrategia metodológica del estudio, seguido de los resultados y la discusión. 


\section{Revisión de literatura}

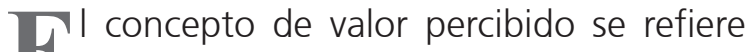
Ca la evaluación global del consumidor de la utilidad de un producto, basada en la percepción de lo que se recibe y de lo que se entrega (Zeithaml, 1988; Kotler y Armstrong 2008); este ofrece diferentes conceptualizaciones (Rodríguez, et al., 2004). Según Kotler y Armstrong (2008), la evaluación realizada del consumidor sobre la diferencia entre todos los beneficios y costos de una oferta de marketing caracteriza todas las definiciones asociadas a este concepto.

Como antecedentes históricos, el valor percibido ha estado asociado a otros dos conceptos importantes para el marketing: la calidad (Zeithaml, 1998) y la satisfacción (Wooddruff y Gardial, 1996). Ostrom y Lacobucci, (1995) lo definen como un lazo entre estos dos tópicos. Gallarza y Gil (1999), refieren las dificultades que rodean la investigación del valor, formulando finalmente un equilibrio entre beneficios y sacrificios. Estos autores realizan una revisión de diferentes enfoques conceptuales del valor percibido, con el fin de darle la relevancia que tiene en las decisiones de compra de los consumidores (Moreno, 2012). Así definir el valor percibido como la evaluación global del consumidor de la utilidad de un producto que implica una evaluación por parte del -cliente/consumidor- sobre sus beneficios y costos (Moreno y Jaramillo, 2012; Gallarza y Saura, 2006).

Según Restrepo (2010), la medición de los beneficios y costos se ha empleado para un dimensionamiento del concepto principalmente, enfocado a las características ofrecidas $y / 0$ recibidas por el mercado. Tal intención hace que los beneficios se estudien a partir de tres niveles: funcionales, concernientes al nivel del desempeño y cumplimiento de la promesa básica enfocada en la eficiencia y la calidad experimentada; los beneficios simbólicos, entendidos como la satisfacción intrínseca que logra el consumidor a partir del entretenimiento, elementos efectivos o de estimulación mental; y los beneficios sociales, referidos a la satisfacción extrínseca a partir del estatus, aceptación de grupo o singularidad. Estos niveles también han sido empleados en otros estudios que asocian el valor percibido con la lealtad del consumidor en suscriptores de telefonía móvil (Karjaluoto, Jayawardhena, Pihlström, y Leppäniemi, 2014) o a las consecuencias de uso del servicio (Pihlstrom, 2008) por lo cual, dicha visión de niveles y/o tipologías del valor percibido orientaran el ejercicio exploratorio.

\subsection{La industria de telefonía móvil en el mercado colombiano}

En el mercado internacional, especialmente el de los jóvenes, es el que reporta mayor nivel de acceso y consumo de la tecnología móvil (Ferrer, 2010). Entre los servicios de telecomunicaciones, el servicio de Telefonía Móvil Celular (TMC) ha registrado los mayores niveles de crecimiento en cobertura y generación de ingresos, tanto para la industria como para la Nación (Oliveros, 2010). En Colombia, un estudio previo realizado por Martínez y Arango (2012) en el ámbito de servicio de mobile marketing, describe que en el mercado de telecomunicaciones, existe un operador dominante que impone tarifas y reglas de comercialización desiguales, siendo la penetración del celular superior al 100\% (p. 136). 
Con respecto a la historia de la telefonía móvil celular en Colombia, se adelantaron a comienzos de 1994, adjudicaciones por medio de una de las licitaciones más importantes del sector de telecomunicaciones del País. La seis empresas de telefonía celular de la época empezaron a fusionarse con el ingreso de capital privado para formar dos empresas con cobertura nacional: Comcel -controlada por América Móvil-, y Movistar -propiedad de Telefónica de España-. Luego a finales de 2003, surgió Colombia Móvil con su marca Ola, que luego se convertiría en Tigo. En noviembre de 2010 nació Uff Móvil, y en el año 2013, surgió la empresa Virgin Mobile. Para comparar el desempeño de estos operadores de telefonía móvil, puede consultarse el último «Informe trimestral de las TIC de 2015» (Mintic, 2015), el cual describe que los proveedores de redes y servicios móviles se encuentran distribuidos de la siguiente manera: Comunicación Celular S.A. -COMCEL S.A- $(53,14 \%)$ : seguido de Colombia Telecomunicaciones S.A. E.S.P., con una participación del 22,95\%, Colombia Móvil S.A. E.S.P. (17,27\%), Virgin Mobile S.A.S. (3,77\%), Uff Móvil S.A.S. $(0,81 \%)$, y los demás proveedores -cuatro- de telefonía móvil, con una participación del 2,06\%. Estos resultados permiten conocer el desempeño de participación de mercado, sin embargo no permiten estimar las razones por las cuales se deben sus efectos, por lo tanto, y a partir de toda la información antes expuesta, el problema de investigación de este documento se concentra en indagar ¿cuál es el valor percibido de los operadores de telefonía móvil, a fin de conocer las características más importantes que pueden influenciar la preferencia y sostenimiento del servicio por parte de sus usuarios. Tal cuestionamiento se considera importante debido al auge de las actividades de marketing que realizan las empresas, la facilidad de comunicación digital de ofertas y servicios, la acelerada apropiación de tecnología y la necesidad de aumentar el conocimiento del consumidor.

\section{Metodología}

$7^{\mid}$diseño de la investigación corresponde Ca un estudio descriptivo relacional (Hernández, Fernández y Baptista, 2006). En correspondencia al marco conceptual antes expuesto, se utilizó la escala de medición PERVAL de valor percibido, que reúne los componentes de beneficios funcionales, simbó-

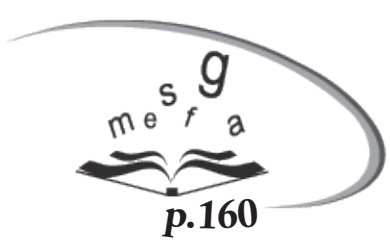
licos y sociales en referencia al costo o sacrificio. Tal escala ha sido tradicionalmente utilizada en la investigación académica por ser multidimensional (Valdés, 2013; Sweeney y Soutar, 2001; Holbrook, 1999;) permite una evaluación a priori del valor percibido por el consumidor (Sales y Saura,
2007), cuyo objetivo es medir los atributos que influyen en la toma de decisiones del consumidor al momento de comparar y elegir un operador móvil. Adicionalmente, la escala PERVAL de Sweeney y Soutar (2001) es hoy en día la escala multidimensional de valor más rigurosa y robusta (Gil y González, 2008).

De acuerdo con los intereses de la investigación y la dinámica de acceso a la tecnología y dispositivos móviles, la población estudiada fueron hombres y mujeres entre los 14 y 35 años de edad. Se aplicaron 376 encuestas, en donde los participantes fueron seleccionados a conveniencia. 
Las preguntas utilizadas para indagar cada una de las dimensiones corresponden a la escala Likert de cinco niveles. Con el fin de mejorar la validez del instrumento, se recurrió a la prueba de fiabilidad Alfa de Cronbach, obteniendo un coeficiente de 0.89 , con lo cual la medición del instrumento es fiable. Posteriormente, se construyó la base de datos utilizando el software estadístico SPSS versión 22.0, para el posterior análisis de resultados.

\section{Resultados}

E I primer grupo de variables analizadas está asociado a las características demográficas de la muestra. Una primera observación tiene que ver con la preeminencia del grupo de edad entre los 20 y 25 años, como parte fundamental del estudio, seguido por el segmento entre 26 y 31 años. Por su parte, la distribución de género concuerda con el estimado nacional donde el $52 \%$ corresponde al género femenino y el $48 \%$ al género masculino.
Con respecto al análisis de las variables de percepción y uso de operadores móviles, se evidencia que el operador Claro ocupa el primer lugar de recordación con un 50,3\%, seguido por Tigo con un 22,5 \% y Movistar con un $18,7 \%$. Los demás operadores no alcanzaron un porcentaje mayor al $10 \%$. Se observan los principales atributos de importancia frente al operador de telefonía móvil, en donde la importancia del atributo señal/conectividad es el más importante (Tabla 1).

Tabla 1. Principales atributos de importancia frente al operador de telefonía móvil

\begin{tabular}{|l|c|c|c|}
\hline & $\begin{array}{c}\text { Principal aspecto de } \\
\text { importancia }\end{array}$ & $\begin{array}{c}\text { Segundo aspecto de } \\
\text { importancia }\end{array}$ & $\begin{array}{c}\text { Tercer aspecto de } \\
\text { importancia }\end{array}$ \\
\hline Señal/conectividad & $76,9 \%$ & $18,8 \%$ & $4,1 \%$ \\
\hline Servicio al cliente & $2,4 \%$ & $17,7 \%$ & $36,7 \%$ \\
\hline Planes y promociones & $8,0 \%$ & $26,1 \%$ & $35,6 \%$ \\
\hline Cobertura & $12,8 \%$ & $37,4 \%$ & $23,4 \%$ \\
\hline
\end{tabular}

Fuente. Elaboración propia de los autores.

A fin de mejorar la comprensión de los operadores de telefonía móvil, se realizó una tabla cruzada para representar la percepción de cada operador, en función de las características motivacionales indagadas (Tabla 2). 
Tabla 2. Tabla cruzada entre servicio de operador actual y aspecto principal que usted destaca del operador móvil

\begin{tabular}{|l|l|c|c|c|c|c|}
\hline \multicolumn{7}{|c|}{ Aspecto principal que usted destaca del operador móvil } \\
\hline Operador & & $\begin{array}{c}\text { Señal / } \\
\text { conectividad }\end{array}$ & $\begin{array}{c}\text { Servicio al } \\
\text { cliente }\end{array}$ & $\begin{array}{c}\text { Planes y } \\
\text { promociones }\end{array}$ & Cobertura & Total \\
\hline Claro & Recuento & 155 & 4 & 8 & 32 & 199 \\
\hline & $\%$ Fila & $77,9 \%$ & $2,0 \%$ & $4,0 \%$ & $16,1 \%$ & $100,0 \%$ \\
\hline Tigo & Recuento & 55 & 1 & 10 & 10 & 76 \\
\hline & $\%$ Fila & $72,4 \%$ & $1,3 \%$ & $13,2 \%$ & $13,2 \%$ & $100,0 \%$ \\
\hline Movistar & Recuento & 65 & 3 & 8 & 5 & 81 \\
\hline & $\%$ Fila & $80,2 \%$ & $3,7 \%$ & $9,9 \%$ & $6,2 \%$ & $100,0 \%$ \\
\hline Une & Recuento & 2 & 0 & 0 & 0 & 2 \\
\hline & $\%$ Fila & $100,0 \%$ & $0,0 \%$ & $0,0 \%$ & $0,0 \%$ & $100,0 \%$ \\
\hline Virgin & Recuento & 12 & 1 & 1 & 1 & 15 \\
\hline & $\%$ Fila & $80,0 \%$ & $6,7 \%$ & $6,7 \%$ & $6,7 \%$ & $100,0 \%$ \\
\hline Orange & Recuento & 0 & 0 & 3 & 0 & 3 \\
\hline & $\%$ Fila & $0,0 \%$ & $0,0 \%$ & $100,0 \%$ & $0,0 \%$ & $100,0 \%$ \\
\hline Total & Recuento & 289 & 9 & 30 & 48 & 376 \\
\hline & $\%$ Total Fila & $76,9 \%$ & $2,4 \%$ & $8,0 \%$ & $12,8 \%$ & $100,0 \%$ \\
\hline
\end{tabular}

Fuente. Elaboración propia de los autores.

Como se puede observar, existen relaciones porcentuales entre el operador y los principales aspectos que lo destacan, con resultados desproporcionados de usuarios en cada operador -155 personas de Claro frente a 65 de Movistar y 55 de Tigo-. El análisis se realiza a partir del criterio de porcentajes fila, en donde se percibe, para cada operador, la característica más predominante, de tal forma que se obtiene que todos se destacan por señal y conectividad, excepto el Orange que sobresale por sus planes y promociones.

En segunda instancia, con respecto a los resultados obtenidos de la aplicación de la escala PERVAL, se presentan los hallazgos organizados por las dimensiones y estadísticos descriptivos resultantes (Tabla 3). 
Tabla 3. Estadísticos descriptivos de las dimensiones del valor percibido aplicado a operadores de telefonía móvil

\begin{tabular}{|c|c|c|c|c|c|c|}
\hline Dimensión & Situación & Media & Mediana & Moda & Asimetría & Curtosis \\
\hline \multirow{4}{*}{$\begin{array}{l}\text { Beneficio } \\
\text { funcional }\end{array}$} & La Señal del operador. & 4,8 & 5 & 5 & $-3,44$ & 15,48 \\
\hline & La cobertura del operador. & 4,5 & 5 & 5 & $-1,92$ & 4,65 \\
\hline & Servicio al cliente. & 4,0 & 4 & 4 & $-0,99$ & 0,94 \\
\hline & Planes y promociones. & 3,9 & 4 & 5 & $-0,86$ & 0,13 \\
\hline \multirow{4}{*}{$\begin{array}{l}\text { Beneficio } \\
\text { emocional }\end{array}$} & $\begin{array}{l}\text { Nivel de agrado hacia la } \\
\text { publicidad de los operadores } \\
\text { móviles. }\end{array}$ & 3,1 & 3 & 3 & $-0,28$ & $-0,62$ \\
\hline & $\begin{array}{l}\text { Nivel de gusto hacia los } \\
\text { operadores móviles. }\end{array}$ & 3,4 & 3 & 3 & $-0,43$ & $-0,20$ \\
\hline & $\begin{array}{l}\text { Nivel de agrado hacia la imagen } \\
\text { de los operadores móviles. }\end{array}$ & 3,4 & 3 & 4 & $-0,50$ & $-0,30$ \\
\hline & $\begin{array}{l}\text { Nivel de felicidad que le ofrecen } \\
\text { los operadores móviles a los } \\
\text { usuarios. }\end{array}$ & 4,0 & 4 & 5 & $-0,94$ & 0,46 \\
\hline \multirow{3}{*}{$\begin{array}{l}\text { Beneficio } \\
\text { social }\end{array}$} & El operador móvil genera Status. & 2,7 & 3 & 1 & 0,18 & $-1,27$ \\
\hline & $\begin{array}{l}\text { Nivel de importancia del operador } \\
\text { móvil en un grupo social. }\end{array}$ & 2,8 & 3 & 3 & $-0,01$ & $-1,07$ \\
\hline & $\begin{array}{l}\text { Nivel de importancia del operador } \\
\text { móvil en el entorno laboral. }\end{array}$ & 3,3 & 4 & 4 & $-0,33$ & $-1,20$ \\
\hline \multirow{3}{*}{$\begin{array}{l}\text { Percepción } \\
\text { de Costo }\end{array}$} & $\begin{array}{l}\text { Los planes que ofrecen los } \\
\text { operadores móviles tienen una } \\
\text { buena relación costobeneficio. }\end{array}$ & 3,1 & 3 & 3 & $-0,15$ & $-0,56$ \\
\hline & $\begin{array}{l}\text { El costo de los planes es un factor } \\
\text { determinante al comparar y elegir } \\
\text { un operador móvil. }\end{array}$ & 4,1 & 4 & 5 & $-1,12$ & 0,59 \\
\hline & $\begin{array}{l}\text { Estoy dispuesto a ahorrar para } \\
\text { adquirir un plan en un operador } \\
\text { móvil. }\end{array}$ & 2,9 & 3 & 3 & $-0,02$ & $-0,91$ \\
\hline
\end{tabular}

Fuente. Elaboración propia de los autores.

Los estadísticos descriptivos presentados anteriormente, permiten demostrar que el atributo con mayor desempeño, es la señal del operador, seguido por la cobertura del operador y el costo. El atributo menos importante fue para «el operador móvil genera status».

Finalmente, con el ánimo de mejorar la comprensión de la dinámica de relaciones entre los atributos, se realizó un análisis factorial como método de reducción de datos, obteniendo cuatro dimensiones del constructo del valor percibido, con un porcentaje de explicación acumulada del $62.2 \%$. El método factorial fue el componente principal, dada su validez como técnica de síntesis de datos (Malhotra, 2011). De acuerdo con lo anterior, se presenta la matriz de componentes principales con rotación varimax, obtenida del análisis de la prueba de esfericidad de Bartlett: $<$ a $0,000 \%$, con $\mathrm{KMO}=$ a 0.788 . 
A continuación se muestran los resultados obtenidos con el análisis factorial correspondiente (Tabla 4). En este caso se han identificado cuatro dimensiones: la dimensión 1 que se ha llamado «beneficios sociales» e integra tres atributos; la dimensión 2 la hemos llamado «beneficios emocionales» que integra seis atributos de dicha cualidad; la dimensión 3 denominada como "percepción de costo» que integra tres atributos; y finalmente la dimensión 4, «beneficios funcionales» que integra dos atributos especialmente importantes en las motivaciones para preferir un operador telefónico móvil.

Tabla 4. Relación por análisis de componentes entre los atributos de valor percibido.

\begin{tabular}{|l|c|c|c|c|}
\hline \multicolumn{1}{|c|}{ Atributos } & \multicolumn{4}{c|}{ Componente } \\
\cline { 2 - 5 } & $\mathbf{1}$ & $\mathbf{2}$ & $\mathbf{3}$ & $\mathbf{4}$ \\
\hline La señal del operador. &, 002 &, 068 &, 012 &, 810 \\
\hline La cobertura del operador. &, 084 &, 029 &,- 059 &, 844 \\
\hline Servicio al cliente. &,- 157 &, 514 &,- 052 &, 474 \\
\hline Planes y promociones. &,- 077 &, 528 &, 112 &, 093 \\
\hline Nivel de agrado hacia la publicidad de los operadores móviles. &, 390 &, 739 &, 055 &,- 009 \\
\hline $\begin{array}{l}\text { Nivel de gusto o agrado de las personas hacia los operadores } \\
\text { móviles. }\end{array}$ &, 344 &, 716 &, 130 &,- 051 \\
\hline Nivel de agrado hacia la imagen de los operadores móviles. &, 403 &, 716 &, 033 &, 003 \\
\hline $\begin{array}{l}\text { Nivel de felicidad que le ofrecen los operadores móviles a los } \\
\text { usuarios. }\end{array}$ &,- 005 &, 649 &, 185 &, 100 \\
\hline El operador móvil genera Status. &, 828 &, 191 &, 093 &, 042 \\
\hline Nivel de importancia del operador móvil en un grupo social. &, 887 &, 140 &, 108 &, 030 \\
\hline Nivel de importancia del operador móvil en el entorno laboral. &, 838 &, 033 &, 151 &,- 027 \\
\hline $\begin{array}{l}\text { Los planes que ofrecen los operadores móviles tienen una buena } \\
\text { relación costo- beneficio. }\end{array}$ &, 121 &, 169 &, 716 &, 024 \\
\hline $\begin{array}{l}\text { El costo de los planes es un factor determinante al comparar y y } \\
\text { elegir un operador móvil. }\end{array}$ &,- 024 &, 077 &, 779 &, 000 \\
\hline $\begin{array}{l}\text { Estoy dispuesto a ahorrar para adquirir un plan en un operador } \\
\text { móvil. }\end{array}$ &, 282 &, 106 &, 675 &,- 113 \\
\hline Varianza total = 62,2. & 29.1 & 13.9 & 10.4 & 8.6 \\
\hline KMO = 0,894; Prueba de esfericidad de Bartlett (p<0,000). & & & & \\
\hline
\end{tabular}

* Análisis factorial de componentes principales con rotación Varimax.

Fuente. Elaboración propia de los autores. 


\section{Discusión}

L a utilización de la escala PERVAL como Jeje de análisis, permitió verificar, como lo señala la literatura, que el valor percibido influye sobre la actitud de los usuarios (Swait \& Sweeney, 2000). Dicha adaptación de escala, conservando el enfoque multidimensional, resultó pertinente para la identificación de los atributos del valor, cuyo conocimiento es central a la administración de factores fundamentales asociados al compromiso y especialmente, a la satisfacción de los usuarios (Ruiz, Gil y Calderón, 2010). El concepto de valor involucrado en el planteamiento metodológico, entendido como la respuesta multidimensional o global a la experiencia de consumo (Ekinci, Dawes y Massey, 2008) encaja con los estudios más recientes sobre esta materia en el ámbito del marketing aplicado.

En esta dinámica, los resultados sugieren que las estrategias de persuasión y fidelización de usuarios basadas en torno al precio, deben estar soportadas en una comunicación que potencie principalmente los factores funcionales como cobertura y señal, articulados a la gestión de factores emocionales y sociales. Cercanía, relaciones familiares y un estilo de vida en línea con las nuevas tecnologías, son elementos que facultan a los operadores de telefonía móvil para aprovechar las motivaciones de los usuarios en la preferencia y sostenimiento de un mismo operador. En esta misma línea, los elementos de orden funcional, los atributos relacionados con la calidad del servicio -señal/conectividad y cobertura-, son los que revisten mayor importancia, seguidos de la oferta comercial en cuanto a planes y promociones. Resulta pertinente para los operadores móviles, indagar por el impacto de la estrategia comercial en la percepción de los usuarios, más allá del simple efecto reactivo en las ventas, reconociendo la importancia de conocer el nivel de satisfacción de sus clientes, traducido en información sobre sus expectativas y percepciones.

Plantear interrogantes del tipo «¿Soy atractivo porque mi oferta es barata o porque los clientes se identifican conmigo?» son aspectos que se deben analizar a la luz de la evidencia presentada en esta investigación. Para el marketing en un contexto digital, debe ser importante la gestión en atributos funcionales -indicadores de calidad-, sin embargo, existen otras dimensiones que aportan valor a la estrategia empresarial. De La Martinière (2008), propone que la gestión estratégica de la marca, en este caso los operadores de telefonía móvil, puede representar un importante diferencial competitivo, pues busca un reconocimiento e identificación que la vuelva única en la mente del consumidor. Al respecto, el caso del operador móvil virtual Virgin Mobile es uno de los más exitosos. Aún cuando su oferta es más económica que los operadores líderes del mercado en Colombia, la compañía se ha enfocado en la generación de un vínculo con el público joven y con ingresos menos estables, pero sin asociar su marca a beneficios funcionales de cobertura o señal, tal como quedó demostrado en los resultados.

Los servicios de comunicaciones móviles, especialmente a través de Internet y el ecosistema de aplicaciones asociadas a estos, trascienden la esfera personal del consumidor convirtiéndose en símbolos con implicaciones culturales, como puede apreciarse en las relaciones emocionales y sociales de los 
consumidores con las marcas de operadores. Tal hallazgo sugiere que, para mantener éxito en la estrategia de marketing incluyendo el ámbito digital, las empresas deben construir y mantener vínculos simbólicos que construyan su propuesta de valor.

Obtener provecho de factores no tradicionales, es decir factores funcionales que consideren los elementos generacionales del consumidor actual, puede aportar a la generación de lealtad (Iglesias y Torres, 2002) más allá de la simple provisión de servicios, debe ser un objetivo fundamental de las estrategias de marketing.

Como limitaciones de la investigación se incluyen el sesgo de respuesta en el operador móvil que tenía cada participante, debido a que una de las marcas de los seis operadores telefónicos móviles indagados, obtuvo más del $50 \%$ de representatividad, por lo cual se recomienda, para próximos estudios, conservar un criterio proporcional.

Finalmente, se espera que este estudio aporte evidencia empírica sobre la importancia y atributos que constituyen el valor percibido, aplicadoaunescenariodelastelecomunicaciones; cabe destacar que el estudio de valor es uno de los temas más populares en la literatura de marketing con lo cual también se legitima el aporte de este documento. 


\section{Referencias bibliográficas}

Oliveros, A. L. (2010). Estado actual del servicio de telefonía móvil en Colombia. Bogotá: Informe para la Contraloría General de la Nación. Dirección de Estudios Sectoriales. Contraloría General de la Republica. Colombia.

Cerezo, J. (2008). Smartphone, Toda la información al alcance de tu mano. Revista Telos.

Ekinci, Y., Dawes, P. y Massey, G. (2008). An extended model of the antecedents and consequences of consumer satisfaction for hospitality services. European Journal of Marketing, 35-68.

Ferrer, A. (2010). Millennials, la generación del siglo XXI. Nueva Revista de Política, Cultura y Arte.

Gallarza, M., y Saura, I. (2006). Desarrollo de una escala multidimensional para medir el valor percibido de una experiencia de servicio. Revista Española de Investigación de Marketing, 25-60.

Gallarza, M., y Gil Saura, I. (1999). Desarrollo de una escala multidimensional para medir el valor percibido de una experiencia de servicio, Revista española de Investigación de Marketing ESIC,

Gil Saura, y González M. (2008). La investigación en valor percibido desde el marketing. Innovar, 18(31), 9-18.

Gómez B., Polo M., Rivera R. (2011). Análisis estratégico del sector de telefonía móvil en Colombia 2005-2010. Documentos de Investigación, Universidad del Rosario, Facultad de Administración, No.108, ISSN: 0124-8219.

Hernández S., Fernández Collado, C., y Baptista L. (2006). Metodología de la investigación. Madrid: McGraw-Hill.
Holbrook, M. (1999). Valor del Consumidor. Un marco para el análisis y la investigación. Londres: Routledge.

International Telecommunication Union. (2014). Key ICT indicators for developed and developing countries and the world (totals and penetration rates). Ginebra Suiza, UIT. Recuperao de http://www.itu. int/en/TU-D/Statistics/Pages/stat/default. aspx consultado el 18 octubre de 2015.

Karjaluoto, H., Jayawardhena, C., Pihlström, M., \& Leppäniemi, M. (2014). Effects of Service Quality, Trust, and Perceived Value on Customer Loyalty: The Case of Mobile Subscribers. In Proceedings of the 2009 Academy of Marketing Science (AMS) Annual Conference. Springer International Publishing, p.179.

Kotler, Philip., y Armstrong, Gary. (2008). Fundamentos de Marketing. México, Pearson Prentice Hall.

Malhotra N. (2011). Investigación de mercados. México: Pearson Educación.

Martínez, O. y Arango, K. (2012). El Mobile Marketing en Colombia, su estado actual y proyección. Revista EAN.

Martínez, R. M., y Jacobo, J. L. G. (2014). Las tecnologías de información y comunicación: su evolución y aplicación en los negocios internacionales. INCEPTUM Revista de Investigación en Ciencias de la Administración, 7(12), 439-466.

MinTIC. (2015). Boletín Trimestral de las TIC, Cifras Primer Trimestre 2015. Bogotá: República de Colombia.

Moreno, J. B., y Jaramillo, J. G. L. (2012). Valor Percibido por el Cliente (VPC), como una herramienta para el análisis competitivo. AD-minister, (6), 96-113. 
Ostrom, A. y lacobucci, D. (1995). Consumer trade- offs and the evaluation of services. Journal of Marketing, 59, 17-28.

Pedraja, I., y Rivera, P. (2002). La gestión de la lealtad del cliente a la organización. Un enfoque de marketing relacional. Economía Industrial, Departamento de Economía y Dirección de Empresas.

Iglesias, M. P., \& Torres, P. R. (2002). La gestión de la lealtad del cliente a la organización: un enfoque de marketing relacional. Economía industrial, (348), 143-153.

Pihlstrom, M. (2008). Perceived Value of Mobile Service Use and Its Consequences. Publications of the Swedish School of Economics and Business Administration. ISBN 9789515559777.

Restrepo, A. (2010). Los jóvenes y sus luchas por el reconocimiento. Revista Nómadas, (32), 179-194.

Revista Dinero (2013). La explosión de las tecnologías móviles. Recuperado de: http://www.dinero.com/edicion-impresa/ especial-comercial/articulo/la-explosiontecnologias-moviles/177019 consultado el 2 de Octubre de 2015.

Rodríguez, R.M., Callarisa, L., Moliner, M.A., y Sánchez, J. (2004). El valor percibido de la compra de un paquete turístico. XVI Encuentros de profesores Universitarios de Marketing. Alicante, 691-696.

Ruelas, A. L. (2010). El teléfono celular y las aproximaciones para su estudio. Comunicación y Sociedad, 143-167.
Ruiz, M., Gil, I., y Calderón, H. (2010). El efecto moderador de la edad en la relación entre el valor percibido, las TIC del minorista y la lealtad del cliente. Cuadernos de Economía y Dirección de la Empresa. 43, 65-92.

Sales, V., y Gil, S, (2007). Valor percibido por el consumidor: una aplicación en la compra de equipamiento para el hogar. Estudios sobre Consumo 82, 35-48

Swait, J., y Sweeney, J. (2000). Perceived value and its impact on choice behavior in a retail setting. Journal of Retailing and Consumer Services 7(2), 77-88.

Sweeney, J. C., y Soutar, G. N. (2001). Consumer perceived value: The development of a multiple item scale. Journal of retailing, 77(2), 203-220.

Valdés, E. (2013). Percepción de las dimensiones de valor de los consumidores en Internet. Magazín Empresarial, 9(22), 49-59.

Vargas L., Rodríguez R., Rojano A., Medina L. y Rivera R., H. (2012). Análisis estratégico del sector teléfonos móviles inteligentes Smartphones. Documentos de investigación de la Facultad de Administración. No. 118, ISSN: 0124-8219

Woodruff B.R. y Gardial F.S. (1996). Know your Costumer: New approaches to Understanding Costumer value and Satisfaction. USA: Blackwell Business.

Zeithaml, V. (1988). Consumer Perceptions of Price, Quality, and Value: A Means-end Model and Synthesis of Evidence. Journal of Marketing 52(3), 2-22. 
